\title{
Cooperative Tracking for Ground and Airborne Passive Sensors in the Presence of Main Lobe Jamming
}

\author{
Qing Sun ${ }^{1}$, Qiliang Zhang ${ }^{1}$ and Qian $\mathrm{Gao}^{2}$ \\ ${ }^{1}$ Air Force Engineering University, Xi'an, 710051, China \\ ${ }^{2}$ Air Force Communication NCO Academy, Dalian, 116600, China
}

\begin{abstract}
Target detection in the presence of mainlobe jamming is a traditional problem for radar signal processing. To solve this problem, methods based on a single array or distributed auxiliary arrays were considered recently. However, the performance is limited by mainlobe resolution or side lobe level, which should be traded off using optimization procedure. To avoid this limitation, a cooperative tracking method for ground and airborne passive sensors is proposed, which can get sufficient resolution for the reason that the airborne sensors can be take near to the target and mainlobe jamming. The target position is fused by extended Kalman filter. The trajectory is optimized to get optimum accuracy. Simulations are then provided to corroborate the theoretical studies.
\end{abstract}

Keywords—mainlobe jamming; sensor data fusion; extended Kalman filter

\section{INTRODUCTION}

Electronic interference against airborne radar are enhanced by using digital radio-frequency memory(DRFM) technology. The jammer with DRFM technique can copy the radar waveform and generate a lot of false targets with various time delays and doppler shifts. It is known that when the jammer is located in the side lobe of radar antenna, the jamming can be cancelled by side lobe cancellation(SLC) technique or side lobe blanking(SLB) technique, but when the jammer is located in main lobe, the false target is very hard to be cancelled, and target detection becomes a problem.

Many target detection methods in the presence of main lobe jamming techniques were proposed up to now. The false target identification technique based on DRFM quantization character analysis technique was proposed in [1], the performance of this technique can be reasonably successful only for signals with rather high SNRs, which will not suit for long range targets. To cancel mainlobe jamming in monopulse radar, main lobe jamming cancellation techniques based on monopulse sumdifference network were studied in [2] to [4]. Adaptive array processing methods for mainlobe jamming cancellation were considered in [5] and [6]. The main lobe jamming cancellation techniques would distort the shape of mainlobe pattern and degrade the gain of the mainbeam on the target direction. To design narrow notch spatial filter, which can keep enough gain on the target, large distributed auxiliary array was employed in [7] and [8], which is designed to suit for wide band and narrow band radar respectively, but the needs of large size auxiliary array would increase cost and volume of the radar system greatly.

To solve this problem, we propose the target detection and tracking method based on the combination of ground based and airborne radar as shown in FIGURE I. For the reason that the airborne radar can be located very near to the observed target, the resolution of the airborne sensor will be much better than that of ground based radar. According to this feature, we design the radar system composed of two ground based radar and two airborne sensors. All the sensors formulate radar beams on the target direction. According to cross location technique, the target can be measured with low accuracy. By sending the UAVs to the expected target position, the airborne sensor will get better accuracy, which will be optimized by particle swarm optimization method.

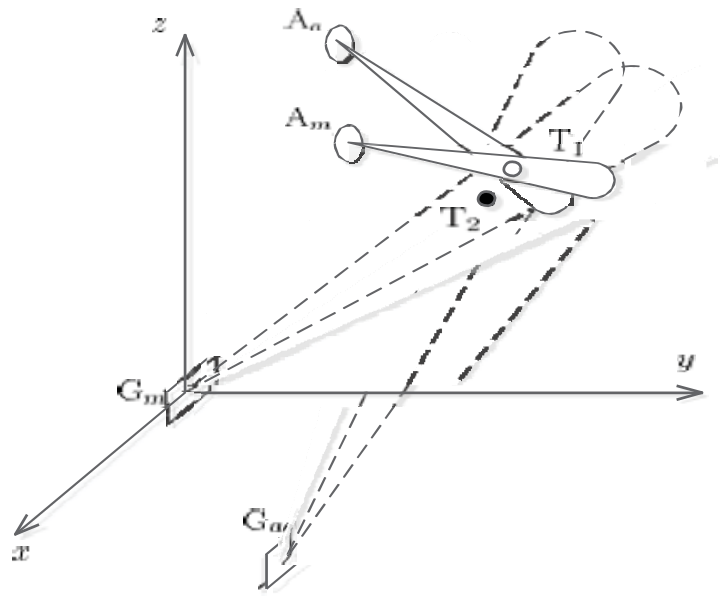

FIGURE I. THE BEAMS OF GROUND BASED RADARS AND AIRBOME SENSORS

Numerical simulation is taken to compare the detection performance with classical methods, which indicated that this method can detect target from complex external environment in the presence of mainlobe jamming.

\section{DATA MODEL}

For ground based radar, the bore sight of two radars on the same target is usually not in the same plane, the measure vector

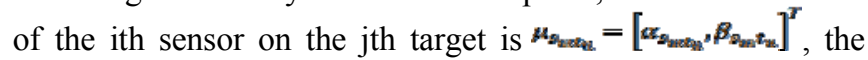


possible target location vector of the ith sensor on the jth target is $v_{s_{m t_{n}}}=\left[\boldsymbol{\alpha}_{m}(x, y, z), \beta_{m}(x, y, z)\right]^{T}$, where

$$
\alpha_{m}=\arcsin \frac{z-z_{s_{m}}}{\sqrt{\left(x-x_{s_{m}}\right)^{2}+\left(y-y_{s_{m}}\right)^{2}+\left(z-z_{s_{m}}\right)^{2}}}
$$

$$
\beta_{m}=\left\{\begin{array}{cr}
\arccos \frac{x-x_{s_{m}}}{\sqrt{\left(x-x_{s_{m}}\right)^{2}+\left(y-y_{s_{m}}\right)^{2}}} & \text { if } y \geq 0 \\
2 \pi-\arccos \frac{x-x_{s_{m}}}{\sqrt{\left(x-x_{s_{m}}\right)^{2}+\left(y-y_{s_{m}}\right)^{2}}} & \text { others }
\end{array}\right.
$$

\section{COOPERATE Tracing}

A. Track Start

The minimum spatial distance method The array coordinates at time tk is $\left(x_{p_{i, k}}, y_{p_{i, k},}, z_{p_{i, k}}\right)$, and the measured angle is $\left(\boldsymbol{\alpha}_{1, k,}, \boldsymbol{\beta}_{i, k}\right)$, then the equations of bore sight of the target are

$$
\frac{x-x_{p_{i, k}}}{\cos \alpha_{i_{2} k} \sin \beta_{i_{1} k}}=\frac{y-y p_{i_{i, k}}}{\cos \alpha_{i_{2} k} \cos \beta_{i_{1} k}}=\frac{z-z_{p_{i, k}}}{\sin \alpha_{i_{1} k}}
$$

Equivalently the line can be expressed as the following equations

$$
\theta_{k} \triangleq\left[\begin{array}{l}
x_{k} \\
y_{k} \\
z_{k}
\end{array}\right]=\left[\begin{array}{l}
x_{0}+v_{x} t_{k} \\
y_{0}+v_{y} t_{k} \\
z_{0}+v_{z} t_{k}
\end{array}\right]=C_{k} \eta
$$

Where $C_{k} \triangleq\left[\begin{array}{ll}I & t_{k} I\end{array}\right]_{3 \times 6 \text { and }} \eta \underline{\underline{ }}\left[x_{0}, y_{0}, z_{0}, v_{x}, v_{y}, v_{z}\right]^{T}$.

Suppose the target coordinate is (Xk, Yk, Zk ), then the foot point of the target can be solved by

$$
\begin{gathered}
\left\{\theta_{k}^{T}-\left[\begin{array}{c}
x \\
y \\
z
\end{array}\right]^{T}\right\}\left[\begin{array}{c}
\cos \alpha_{i, k} \sin \beta_{i, k} \\
\cos \alpha_{i, k} \cos \beta_{i, k} \\
\sin \alpha_{i, k}
\end{array}\right]=0 \\
v_{f_{i, k}}=\left[\begin{array}{l}
x_{f i, k} \\
y_{f_{i, k}} \\
z_{f_{i, k}}
\end{array}\right]=A_{k}^{-1} b_{k}
\end{gathered}
$$

Where

$$
A_{k}=\left[\begin{array}{ccc}
\cos \beta_{i, k} & -\sin \beta_{l, k} & 0 \\
\sin \alpha_{i, k} & 0 & -\cos \alpha_{i, k} \sin \beta_{i, k} \\
\cos \alpha_{i, k} \sin \beta_{i, k} & \cos \alpha_{i, k} \cos \beta_{i, k} & \sin \alpha_{i, k}
\end{array}\right]
$$

The determination of matrix $A_{k}$ is $\sin \beta_{t, k}$

$$
\begin{aligned}
& b_{k}=\left[\begin{array}{c}
x_{p_{i, k}} \cos \beta_{i, k}-y_{p_{i, k}} \sin \beta_{i, k} \\
x_{p_{i, k}} \sin \alpha_{i, k}-z_{p_{i, k}} \cos \alpha_{i, k} \sin \beta_{i, k} \\
x_{k} \cos \alpha_{i, k} \sin \beta_{i, k}+y_{k} \cos \alpha_{i, k} \cos \beta_{i, k}+z_{k} \sin \alpha_{i, k}
\end{array}\right] \\
& =c_{k}+D_{k} \theta_{k}=c_{k}+D_{k} c_{k} \eta
\end{aligned}
$$

The variable vector is

$$
\begin{gathered}
c_{k} \triangleq\left[\begin{array}{c}
x_{p_{i, k}} \cos \beta_{i, k}-y_{p_{i, k}} \sin \beta_{i, k} \\
x_{p_{i, k}} \sin \alpha_{i, k}-z_{p_{i, k}} \cos \alpha_{i, k} \sin \beta_{i, k} \\
0
\end{array}\right] \\
D_{k}=\left[\begin{array}{ccc}
0 & 0 & 0 \\
0 & 0 & 0 \\
\cos \alpha_{i, k} \sin \beta_{i, k} & \cos \alpha_{i, k} \cos \beta_{i, k} & \sin \alpha_{i, k}
\end{array}\right]
\end{gathered}
$$

The distance between the foot point $\left(x_{f_{i, K^{\prime}}} y_{f_{i, K^{\prime}},} z_{f_{i, k}}\right)$ and the expected $\operatorname{dot}\left(\boldsymbol{X}_{k}, \boldsymbol{y}_{k}, \boldsymbol{z}_{k}\right)$ is

$$
d_{k}=\sqrt{\left(x_{f_{i, k}}-x_{k}\right)^{2}+\left(y_{f_{i, k}}-y_{k}\right)^{2}+\left(z_{f_{i, k}}-z_{k}\right)^{2}}
$$

Then the summation of the squared error is

$$
\begin{aligned}
& \xi=\sum_{i=1}^{P_{i}} \sum_{k=1}^{K}\left(x_{k}-x_{k}\right)^{2}+\left(y_{k}-y_{k}\right)^{2}+\left(z_{k}-z_{k}\right)^{2} \\
& =\sum_{i=1}^{P_{i}} \sum_{k=1}^{K}\left(v_{k}-\theta_{k}\right)^{T}\left(v_{k}-\theta_{k}\right)
\end{aligned}
$$

Then the position of the jamming can be get by solving the optimization model

$$
\min \xi
$$

Letting the derivative of $\xi$ be zero, we have

$$
\begin{aligned}
\frac{\partial \partial_{\xi}}{\partial \eta_{T}} & =\sum_{i=1}^{P_{i}} \sum_{k=1}^{K} 2 \frac{\partial\left(v_{k}-\theta_{k}\right)^{T}}{\partial \eta}\left(v_{k}-\theta_{k}\right) \\
& =\sum_{i=1}^{P_{t}} \sum_{k=1}^{K} 2 \frac{\partial\left[\theta_{k}^{-1}\left(c_{k}+D_{k} C_{k} \eta\right)-c_{k} \eta\right]^{T}}{\partial \eta}\left(v_{k}-\theta_{k}\right)
\end{aligned}
$$




$$
=\sum_{t=1}^{P_{i}} \sum_{k=1}^{K} 2 C_{k}^{T}\left[D_{k}^{T}\left[\mathcal{A}_{k}^{-1}\right]^{T}-I\right]\left(v_{k}-\theta_{k}\right)
$$

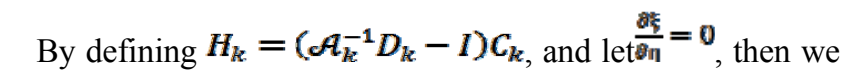
get

$$
\sum_{i=1}^{P_{i}} \sum_{k=1}^{K} 2 H_{k}^{T}\left[\mathcal{A}_{k}^{-1} \mathcal{c}_{k}+H_{k} \eta\right]=0
$$

and

$$
\begin{gathered}
\sum_{i=1}^{P_{t}} \sum_{k=1}^{K} H_{k}^{T} H_{k} \eta=-\sum_{i=1}^{P_{i}} \sum_{k=1}^{K} H_{k}^{T} \mathcal{A}_{k}^{-1} c_{k} \\
\eta=-\left(\sum_{i=1}^{P_{t}} \sum_{k=1}^{K} H_{k}^{T} H_{k}\right)^{-1} \sum_{i=1}^{P_{i}} \sum_{k=1}^{K} H_{k}^{T} \mathcal{A}_{k}^{-1} c_{k}
\end{gathered}
$$

\section{B. UAV Trajectory Optimization}

In order to optimize the trajectory of the UAVs, the target location accuracy was selected as the objective function by traditional methods. The accuracy can be measured by the onesigma ellipsoid of the estimated target position. Under regularity conditions, the PDF of the ML estimate $\theta^{\wedge}$ converges to Gaussian PDF with mean $\theta$ and covariance equal to the Cramer-Rao bound

$$
\mathrm{p}(\hat{\theta})=\frac{e^{-\frac{1}{2}[\hat{\theta}(\hat{R})-\theta(k)]^{T}}}{\sqrt{(2 \pi)^{\mathbb{N}} \theta\left|F_{\theta}\right|}}
$$

where

$$
\begin{gathered}
\mu=\left[\alpha_{1}, \beta_{1}, \alpha_{1}, \beta_{1}\right]^{T} \\
\theta=[x, y, z]^{T}
\end{gathered}
$$

The Jacobian matrix is defined as

$$
J=\frac{\partial \mu^{T}}{\partial \theta}
$$

The Cramer-Rao Bound of the coordinates is

$$
F_{\theta}=\left[J \Sigma_{\mu}^{-1} J^{T}\right]^{-1}
$$

Then the volume of one-sigma ellipsoid corresponding to the three-dimensional case can be calculated as

$$
\begin{aligned}
V_{1 \sigma} & =\iiint_{p(\hat{\boldsymbol{\theta}}) \geq \frac{e^{-\frac{1}{2}}}{\sqrt{(2 \pi)^{N}}}} \mathrm{~d} V \\
& =\iiint_{\frac{x^{2}}{\sigma_{\boldsymbol{x}}^{2}}+\frac{y_{\boldsymbol{\theta}} \mid}{\sigma_{\boldsymbol{y}}^{2}}+\frac{z^{2}}{\sigma_{\boldsymbol{z}}^{2}} \leq 1} \mathrm{~d} V \\
& =\frac{4}{3} \sigma_{\boldsymbol{x}} \sigma_{\boldsymbol{y}} \sigma_{\boldsymbol{z}} \\
& =\frac{4}{3}\left|F_{\boldsymbol{\theta}}\right|
\end{aligned}
$$

For traditional methods, $\mathrm{V}_{1 \sigma}$ is modeled as a function of the trajectory, which is optimized by particle swarm optimization algorithm at each sample time.

In the practical case, this method is only local optimum solution at each sampled point. However, they are not global optimized. Hence we propose a trajectory programming method based on proportional navigation method, which will get more flat trajectory than traditional method. This navigation method will arrive at the target much earlier than traditional methods. The principle of the navigation method is shown in FIGURE II.

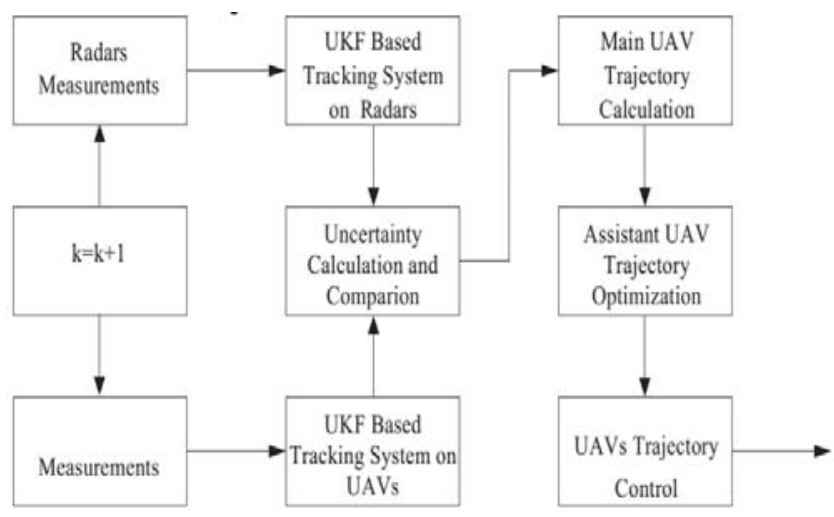

FIGURE II. SCHEMA OF TARGET TRACKING SYSTEM

\section{EKF Algorithm}

The position of the target can be traced by the following EKF algorithm.

Status variable prediction

$$
\hat{X}(k+1 \mid k)=f[k, \hat{X}(k \mid k)]
$$

Covariance prediction

$$
\mathrm{P}(\mathrm{k}+1 \mid k)=f_{X}(k) P(k \mid k) f_{X}^{T}(k)+Q(k)
$$

Measure value prediction

$$
\hat{Z}(k+1 \mid k)=h_{X}[k+1, \hat{X}(k+1 \mid k)](k+1) P(k+1 \mid k)
$$

The covariance matrix with respect to measure value 


$$
S(k+1)=h_{X}(k+1) P(k+1 \mid k) h_{X}^{T}(k+1)+R(k+1)
$$

The filter gain

$$
\mathbf{K}(k+1)=P(k+1 \mid k) h_{X}^{T}(k+1) S^{-1}(k+1)
$$

Status update equation

$$
\begin{aligned}
& \hat{X}(k+1 \mid k+1)=\hat{X}(k+1 \mid k)+K(k+1)\{Z(k+1) \\
& -k[k+1, \hat{X}(k+1 \mid k)]\}
\end{aligned}
$$

Covariance update equation

$$
\begin{gathered}
P(k+1 \mid k+1)=\left[I-K(k+1) h_{X}(k+1)\right] P(k+1 \mid k) \\
{\left[I+K(k+1) h_{X}(k+1)\right]^{T}-K(k+1) R(k+1) K^{T}(k+1)(30)}
\end{gathered}
$$

\section{NumERICAL SimUlation}

To show the performance of the proposed method, numerical simulations are performed as follows.

- Trajectory optimization for two UAVs.

- Target estimation error of two UAVs.

The simulations are performed on a cooperated ground based and airborne radar system.

The coordinates of ground radars are $(0,0,0) \mathrm{km}$ and $(0,20$, $0) \mathrm{km}$. The original position of target is $(100,10,10) \mathrm{km}$, and its velocity is $(-100,100,0) \mathrm{m} / \mathrm{s}$.

The optimized trajectory is shown in FIGURE III. It can be seen that the proposed trajectory is much more flat than other method.

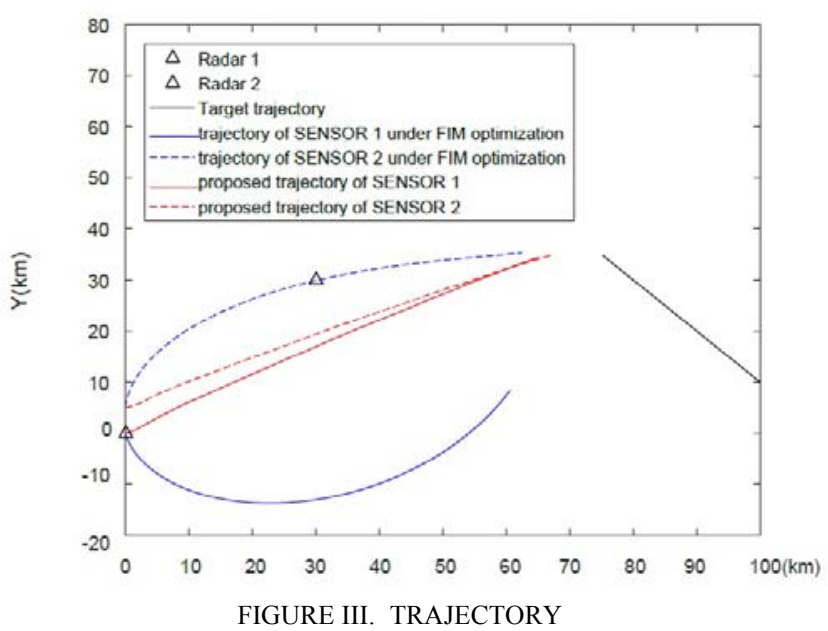

FIGURE IV shows the target position estimation accuracy of the proposed method and method. The four sub figures are the accuracy of $x, y, z, R$ respectively. The red and blue curve represents the accuracy of the proposed method and other method respectively. It is obvious that the proposed method can get higher accuracy at each dimension and the total slant-range direction.

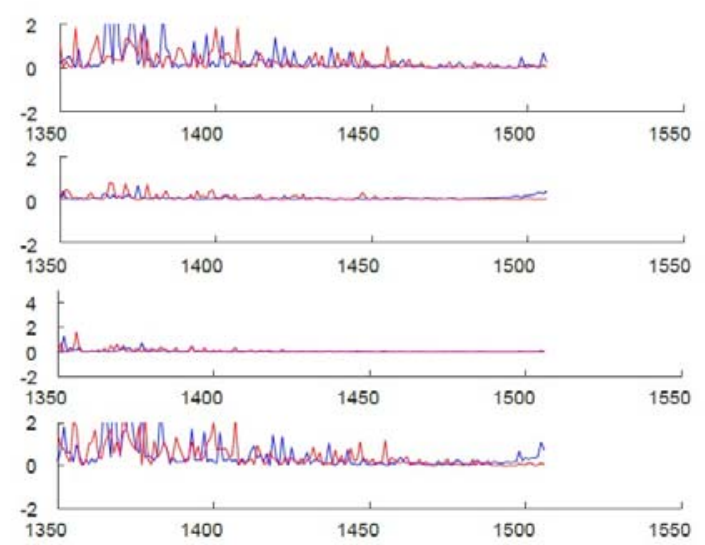

FIGURE IV. TARGET POSITION TRACKING ERROR(')

\section{CONCLUSION}

In this paper, a passive target tracing method based on ground based and airborne sensors is proposed, which can get much higher accuracy than traditional methods.

\section{REFERENCES}

[1] P.C.J. Hill, V.Truffert, "Statistical processing techniques for detecting DRFM repeat-jam radar signals," IEE Colloquium on Signal Processing Techniques for Electronic Warfare, pp. 1-6, Jan. 1992.

[2] K. B. Yu and D. J. Murrow, "Adaptive digital beamforming for Angle Estimation in Jamming,” IEEE Trans. Aerosp. Electron. Syst, vol. 37, no. 2, pp. 508-23, Apr. 2001.

[3] S. H. Moon,D. S. Han, H. S. Oh and M. J. Cho, "Monopulse angle estimation with constrained adaptive beamforming using simple mainlobe maintenance technique," IEEE Military Communications Conference, vol. 2, no. 2, pp. 1365-9, Oct. 2003.

[4] R. F. Li,C. Rao,L. Y. Dai and Y. L. Wang, "Combining sum-difference and auxiliary beam for adaptive monopulse in jamming," Journal of systems Engineering and Electronics,vol. 24, no. 3, pp. 372-381, Jun. 2013.

[5] U. Nickel, "Monopulse estimation with adaptive arrays," IEE Proceedings of Radar and Signal Processing, vol. 140, no. 5, pp. 303 308, Oct. 1993.

[6] S. P. Applebaum, D. J. Chapman, "Adaptive arrays with mainbeam constraints," IEEE Trans. on AP, vol.AP-24, pp. 650-662, Sep. 1976.

[7] R. L. Fante,R. M. Davis and T. P. Guella,"Wideband cancellation of multiple mainbeam jammers," IEEE Transactions on Antennas and Propagation, vol. 44, no. 10, pp. 1402-1413, Oct. 1996.

[8] X. P. Yang, P. L. Lin, T. Zeng and T. K. Sarkar,"Applying auxiliary array to suppress mainlobe interference for ground-based radar," IEEE Antennas and Wireless Propagation Letters, vol. 12, pp. 433-436, Mar. 2013.

[9] H. L. Van Trees, Optimum Array Processing-Part IV of Detection, Estimation, and Modulation Theory. New York, NY, USA:Wiley, 2002.

[10] Y. C. Eldar and G. Kutyniok, Compressed Sensing:Theory and Applications. New York, NY, USA:Cambridge University Press, 2012.

Zhen Liu, Xizhang Wei and Xiang Li,"CS-based moving target detection in random PRI radar," IEEE International Geoscience and Remote Sensing Symposium, pp. 7476-7479, Jul. 2012. 